\title{
Environmental Effects on the Kinematics of Virgo Cluster Galaxies
}

L. Chemin ${ }^{1,2}$, V. Cayatte ${ }^{2}$, C. Balkowski ${ }^{2}$, P. Amram ${ }^{3}$, M. Marcelin ${ }^{3}$, O. Garrido ${ }^{3}$, J. Boulesteix ${ }^{3}$, C. Carignan ${ }^{1}$, A. Boselli $^{3}$, B. Vollmer ${ }^{4}$, C. Adami $^{3}$, O. Hernandez ${ }^{1,3}$

${ }^{1}$ Dept. de Physique, Université de Montréal, C.P. 6128, Succ.

Centre-ville, Montréal, Qc, H3C3J7, Canada

${ }^{2}$ GEPI, Observatoire de Paris, 5 place J. Janssen, 92195 Meudon, France

${ }^{3}$ Observatoire Astronomique de Marseille-Provence, 2 Pl. Le Verrier, 13248 Marseille, France

${ }^{4}$ CDS, Observatoire de Strasbourg, 11 rue de l'Université, 67000 Strasbourg, France

\begin{abstract}
We present results from an ongoing survey of the ionized gas kinematics in Virgo cluster spiral galaxies using Fabry-Perot (FP) interferometry. Our goal is to study the environmental effects on galaxy evolution in the Virgo cluster. We report here on the $\mathrm{H} \alpha$ distribution map and velocity field of NGC 4438, an interacting galaxy near the centre of the cluster.
\end{abstract}

\section{Introduction}

Environmental effects play an important role on the evolution of galaxies in clusters. Different mechanisms are invoked to explain the morphology segregation observed in clusters like tidal interactions between galaxies and ram pressure stripping exerted by the intra-cluster medium on disc galaxies (see Combes 2003, van Gorkom 2003 and references therein).

We are completing a survey of Virgo cluster spiral galaxies by Fabry-Perot observations (Chemin 2003) in order to determine the role of tidal interactions and ram-pressure stripping (ICM-ISM interactions) on their ionized gas kinematics. Observed data-cubes will be compared with the results of $3 \mathrm{D}$ simulations of ICM-ISM and/or tidal interactions (e.g. Vollmer 2003).

We will also investigate the influence of the environmental effects on the dark matter distribution by comparing mass models with the results of GHASP, a FP survey of local isolated galaxies (Garrido et al. 2002).

\section{Observations}

We observe galaxies for which exist deep $\mathrm{H} \alpha$ images (Koopmann et al. 2001, Gavazzi et al. 2002) and which present perturbations in their rotation curves as derived from long-slit measurements (Rubin et al. 1999). The survey spans all 

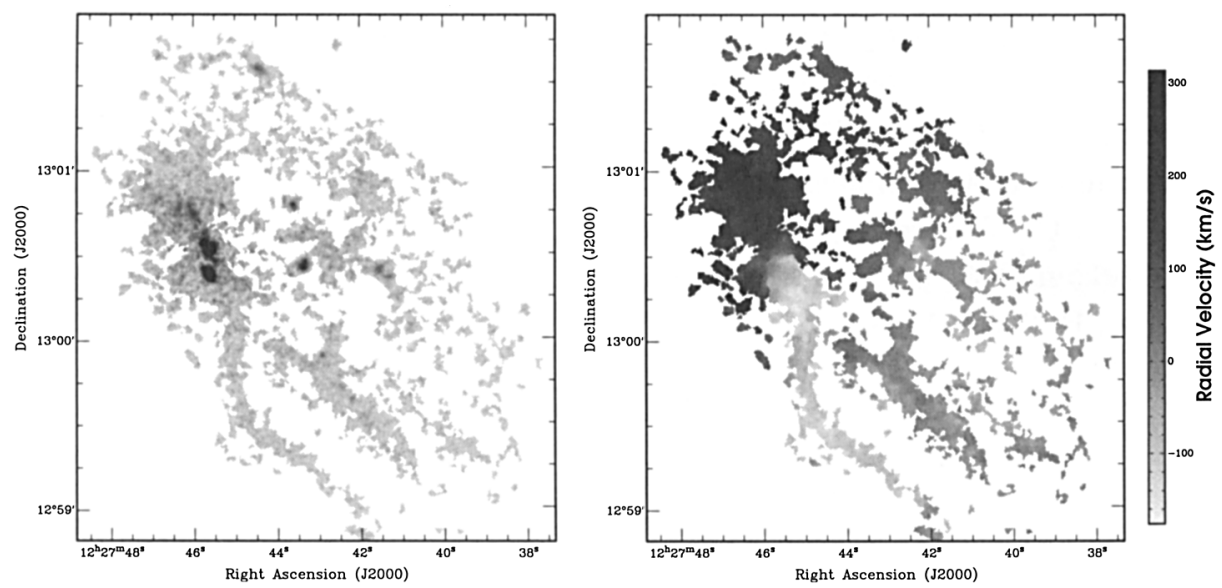

Figure 1. $\mathrm{H} \alpha$ monochromatic map (left) and velocity field (right) of NGC 4438 as obtained by FP observations.

morphological types from S0a to Irregulars. We have already observed 25 objects which are distributed over the whole cluster, with a concentration around M87 in the cluster core where the environmental effects are expected to be stronger. The observations take place at the $1.6 \mathrm{~m}$ Observatoire du mont Mégantic (Canada), 1.93m Observatoire de Haute-Provence (France), 3.6m Canada-France-Hawaii and $3.6 \mathrm{~m}$ ESO telescopes, equipped with a scanning FP interferometer coupled with a photon-counting camera of very high sensitivity (FaNTOmM and GHASP instruments, Gach et al. 2002). The observation of NGC 4438 (Fig. 1) has been done on April 2002 at the 3.6m ESO telescope. The field of view is $3.6^{\prime}$ and the spatial sampling is $0.42^{\prime \prime}$. The spectral step and the total exposure time were $16 \mathrm{~km} / \mathrm{s}$ and $9360 \mathrm{~s}$ respectively.

\section{Results}

Figure 1 (left) presents the H $\alpha$ emission map of NGC 4438, a highly inclined disc located near the cluster core. It shows bright HII regions which are associated to the galactic disc and to the nuclear outflow recently evidenced by Kenney \& Yale (2002). Diffuse filaments along which are observed a few brighter clumps are seen on the western side of the disc. The right panel of the figure presents the complex velocity field of NGC 4438 (Chemin et al., in prep.). Both fields indicate strong signs of perturbations, probably induced by an ICM-ISM interaction and/or a tidal interaction with a companion.

\section{References}

Chemin, L. 2003, PhD Thesis, Université Paris VI

Combes, F. 2003, astro-ph/0308293

Gach, J.-L., et al. 2002, PASP, 114, 1043 
Garrido, O., et al. 2002, A\&A, 387, 821

Gavazzi, G., et al. 2002, A\&A, 386, 114

Kenney, J. D. P., \& Yale E. E. 2002, ApJ, 567, 865

Koopmann, R. A., Kenney, J. D. P., \& Young, J. 2001, ApJS, 135, 125

Rubin, V. C., Waterman, A. H., \& Kenney, J. D. P. 1999, AJ, 118, 236

van Gorkom, J. H. 2003, astro-ph/0308209

Vollmer, B. 2003, A\&A, 398, 525 